\title{
Starfish-inspired Ultrasound Ciliary Bands for Microrobotic Systems
}

\section{Cornel Dillinger}

ETH Zurich https://orcid.org/0000-0002-1734-5713

\section{Nitesh Nama}

The Pennsylvania State University

Daniel Ahmed ( $\sim$ dahmed@ethz.ch )

ETH Zurich https://orcid.org/0000-0002-0224-5293

\section{Article}

Keywords: Bioinspired, Soft Robots, Cilia, Propulsion, Acoustics

Posted Date: January 7th, 2021

DOl: https://doi.org/10.21203/rs.3.rs-138082/v1

License: (1) This work is licensed under a Creative Commons Attribution 4.0 International License. Read Full License

Version of Record: A version of this preprint was published at Nature Communications on November 9th, 2021. See the published version at https://doi.org/10.1038/s41467-021-26607-y. 


\title{
Starfish-inspired Ultrasound Ciliary Bands for Microrobotic Systems
}

\author{
Cornel Dillinger ${ }^{1}$, Nitesh $\mathrm{Nama}^{2}$, and Daniel Ahmed ${ }^{1 *}$ \\ ${ }^{1}$ Acoustic Robotics Systems Lab, Department of Mechanical and Process Engineering, ETH Zurich, \\ Switzerland \\ ${ }^{2}$ Department of Surgery, University of Michigan, Ann Arbor, Michigan, USA \\ *Correspondence and requests for materials should be addressed to D.A. (e-mail: dahmed@ethz.ch)
}

\begin{abstract}
Cilia are short, hair-like appendages ubiquitous in various biological systems, which have evolved to manipulate and gather food in liquids at regimes where viscosity dominates inertia. Inspired by these natural systems, synthetic cilia have been developed and cleverly utilized in microfluidics and microrobotics to achieve functionalities such as propulsion, liquid pumping and mixing, and particle manipulation. In this article, we present the first demonstration of ultrasound-activated synthetic ciliary bands that mimic the natural arrangements of ciliary bands on the surface of starfish larva. Our system leverages nonlinear acoustics at microscales to drive bulk fluid motion via acoustically actuated small-amplitude oscillations of synthetic cilia. By arranging the planar ciliary bands angled towards (+) or away (-) from each other, we achieve bulk fluid motion akin to a flow source or sink. We further combine these flow characteristics with a novel physical principle to circumvent the scallop theorem and realize acoustic-based propulsion at microscales. Finally, inspired by the feeding mechanism of a starfish larva, we demonstrate an analogous microparticle trap by arranging + and - ciliary bands adjacent to each other.
\end{abstract}

Keywords: Bioinspired | Soft Robots | Cilia | Propulsion | Acoustics 


\section{Introduction}

Cilia are short, hair-like appendages present in numerous biological living systems. They can be found on the surfaces of many organisms, including algae and invertebrate larvae, which are naturally evolved to manipulate and gather food in liquids, where viscosity dominates inertia. Ciliated surfaces are also present in most mammals' respiratory tracts, where they trap and move particulates towards the nostrils. Ciliated surfaces are further manifested in the Fallopian tube to transport the ovum towards the uterus. Recent studies on the larval stages of marine invertebrates, such as Patiria miniate (starfish), have elicited that these invertebrates can adjust the orientation of cilia in their ciliary bands (i.e., densely packed cilia) to control the direction of liquid flow; i.e. an analogous fluid source and sink are developed for their propulsion and feeding mechanisms ${ }^{1}$. Inspired by nature's cilia and their functions, engineered synthetic cilia and ciliary bands are of great interest for lab-on-chip devices and microrobotic systems. In particular, they promise solutions for many fundamental functions including propulsion, liquid pumping and mixing, and particle manipulation- all difficult to realize at microscale due to a lack of inertia.

Artificial cilia are commonly driven by external fields, such as electric, magnetic, light, and pressure fields. Magnetically-driven cilia, in particular, have become popular due to their relatively simple and easy operation. For example, the motion of artificial cilia has been demonstrated with a handheld magnet applied to arrays of composite magnetic-polymeric nanorod arrays ${ }^{2}$. In another method, magnetism-based self-assembled cilia are first formed in a chain through the dipole-dipole interaction of superparamagnetic particles immersed in liquid, and subsequently rotated via a rotating magnetic field ${ }^{3}$. These and similar concepts have been implemented for fluid pumping and particle transport ${ }^{4}$. In addition, $1 \mathrm{D}$ and 2D arrangements of magnetic cilia have been shown to exhibit metachronal waves and used for various applications in fluid and particle transport and soft robotics ${ }^{5,6}$. In alternative approaches, electrostaticallyactivated cilia are created with metal-coated polymeric films for mixing applications at low Reynolds number; light-driven cilia are fabricated using azo-benzene doped liquid crystals $^{7}$; and cilia containing multiple pneumatically-controlled actuators ${ }^{8}$ are developed to generate propulsion in liquid ${ }^{9}$.

While several different approaches have been developed to exploit artificial cilia for biomedical applications, acoustically-activated cilia have received little attention - a surprising trend given the widespread use of ultrasound in biomedical and clinical systems. In particular, while the acoustic vibration of individual synthetic cilia has been employed for liquid pumping ${ }^{9}$, the interaction of multiple cilia in close proximity i.e. in ciliary bands (as observed in biological systems), remains largely unexplored. Inspired by the natural arrangements of the cilia on the surface of starfish larva, we developed ultrasound-activated ciliary bands. Notably, cilia and 
ciliary bands activated by ultrasound are particularly attractive because ultrasound is safe to most biological systems, non-invasive, penetrates deep in the body of an animal model, and is widely used in clinical settings. Ultrasound-based ciliary bands promise to be an extremely versatile tool for various lab-on-chip applications and developing micro- and nanorobots for in vivo applications. This article presents the first demonstration of ultrasound-activated synthetic ciliary bands to realize multi-functional microrobotic systems. When our synthetic polymeric ciliary bands are exposed to an acoustic field, they undergo small-amplitude oscillation and execute a bulk liquid flow. When the ultrasound-actuated planar ciliary bands are angled toward each other $(+)$, the fluid is pushed away from the surface on which the bands are housed, mimicking a flow source. In contrast, when the cilia are angled away from each other (-), the liquid is forced in toward the surface, mimicking a flow sink.

Achieving externally-controlled propulsion at the microscale represents another significant challenge to realizing micro- and nanorobotic manipulation systems for in vivo use. The well-known scallop theorem states that the reciprocal motion of a two-state motor submerged in a Newtonian liquid at low Reynolds number results in no net motion. To date, only a few studies have been carried out that used reciprocal motion for propulsion at the microscale. One example is the "micro-scallop," which uses a magnetic field oscillating at $0.5 \mathrm{~Hz}$ to perform reciprocal-motion-based propulsion in non-Newtonian shear-thinning and -thickening gels ${ }^{10}$. In another study, periodic motion of a rigid body introduced nonlinearities through interfacial deformation, thus generating propulsion under low-Reynolds number flow fields ${ }^{11}$. In this work, we leveraged nonlinear acoustics in conjunction with a source/sink arrangement of ciliary bands to develop a new physical principle of propulsion for acoustic-based microrobots. Specifically, we present a unique approach to micro-propulsion that overcomes the scallop theorem by using high-frequency reciprocal oscillation of ciliary bands to locally induce considerable inertial forces and consequent propulsion. Thus, we render the contingency of the scallop theorem invalid by introducing inertia in an otherwise viscous dominated flow regime ${ }^{12}$. Finally, by arranging + and - ciliary bands, i.e. a source and sink, adjacent to each other, we demonstrate a microparticle trap mimicking the feeding mechanism of starfish larva.

\section{Results}

\section{Bioinspired Ciliary Bands}

Inspired by the remarkable natural arrangements of ciliary bands on the surface of invertebrate larvae, we developed new designs for ultrasound-based ciliary bands that leverage the same physical principles. To validate our concept, we fabricated "+" and "-" arrangements of cilia using ultraviolet (UV) photopolymerization method and performed experiments to characterize their behaviour in an acoustic field. The UV photopolymerization method was developed under an inverted microscope. In short, masks containing the ciliary band designs were placed at the 
field stop of the microscope. UV light passes through a 20x objective and polymerized the polyethylene glycol and photoinitiator solution sandwiched between two glass slides (Fig. S1). The fabricated + and - ciliary bands comprised two to eight cilia on each side. Each cilium had a length, base thickness, and height of $\mathrm{L} \approx 100 \mu \mathrm{m}, \mathrm{W} \approx 15-35 \mu \mathrm{m}$, and $\mathrm{H} \approx 50 \mu \mathrm{m}$ (Fig. S2), respectively, and as a set were arranged in series with separation of $20-40 \mu \mathrm{m}$. After fabrication, the ciliary bands were placed in an acoustic chamber filled with liquid solution containing tracer particles. A piezo transducer, which generated the acoustic field, was bonded next to the acoustic chamber and connected to an electronic function generator (Fig. S3). The entire setup was mounted on an inverted microscope, and experimental results were captured using light-sensitive and high-speed cameras.

Over the course of the experiment, the acoustic field's excitation frequency was modulated from $20-100 \mathrm{kHz}$ while maintaining an applied peak-to-peak voltage of $1-25 \mathrm{~V}_{\mathrm{PP}}$. When exposed to such an acoustic field, a ciliary band undergoes small-amplitude oscillations (Fig. S4), which result in a time-averaged steady flow field characterized by a pair of counterrotating vortices in the surrounding liquid, also referred as acoustic streaming ${ }^{13}$. This acoustic streaming is driven by the dissipation of acoustic energy flux inside the fluid system that occurs, in general, both within and outside of the viscous boundary layer. However, for devices where the characteristic dimensions are much smaller compared to the acoustic wavelength $(L \ll \lambda)$, the acoustic streaming is primarily driven by the viscous dissipations within the boundary layer. For the cilia system considered in this work, the thickness of viscous boundary layer can be computed as $\delta=\sqrt{v /(\pi f)}=\sim 1.8-4.0 \mu \mathrm{m}$, where $v \approx 10^{-6} \mathrm{~m}^{2} / \mathrm{s}$ is the kinematic viscosity of water at room temperature, and $f=20-100 \mathrm{kHz}$ is the excitation frequency. The viscous dissipations in this thin boundary layer drive the outer bulk streaming motion. This streaming profile is determined by the arrangement of the ciliary array relative to the normal axis of the underlying bulk surface. Referring to Fig. 1a insets, a + ciliary band comprises a pair of ciliary arrays that face each other and causes the liquid to flow away on a plane perpendicular to the centre of the ciliary band, analogous to a fluid source. Conversely, a - ciliary band consists of two ciliary arrays oriented away from each other and directs the streamlines of the liquid flow to the centre of the band, analogous to a fluid sink. Fig. $\mathbf{1 b}$ represents the complex flow profile of a bioinspired microrobot by combining + and - ciliary bands.

To understand the behaviour of a + ciliary band, we first looked at particle transport in the right half array of the + ciliary band when exposed to ultrasound. The tracer particles, as indicated by red, green, and blue trajectories in Fig. 2a, rapidly hop from the tip of one cilium to another, with particles achieving velocities as high as $10 \mathrm{~mm} / \mathrm{s}$ when they approach the tip. The ciliary array's direction dictates the flow direction, i.e. the flow is guided from right-to-left along 
the right ciliary array (Fig. $\mathbf{2 b}$ and Movie S1). The rapid motion of particles along that array produces a clockwise $(\mathrm{CW})$ vortex in the right half of the ciliary band; similar fluid flow along the left half produces a counter-clockwise (CCW) vortex. Thus, an analogous acoustic source is formed due to build-up of the two counter-rotating vortices, producing an outward flow at the centre of the + ciliary band, as shown in Fig. 3a. Fig. 3b likewise illustrates the liquid flow field near a - ciliary band. In contrast to Fig. 3a, the flow field for a - ciliary band is analogous to a fluid sink and produces an inward flow at the centre (see also Movie S2). In a control experiment, when a straight ciliary array was exposed to an acoustic field, we observed no significant particle transport or fluid flow from one cilium to another (Fig. S5 and Movie S3). Instead, the tracer particles were trapped in small vortices around each cilia tip. Therefore, angulated ciliary array is responsible for the tangential streaming in the direction of the cilia tips, which is critical to developing the acoustic analogous source and sink.

The strength of the flow produced from + and - ultrasound ciliary bands is determined by the intensity of the ambient acoustic field, which is controlled by adjusting the voltage applied to the piezoelectric transducer. Specifically, the streaming is driven by force and mass sources that depend quadratically on the first-order pressure and velocity, which in turn, depend linearly on the applied displacement amplitude ${ }^{14}$. Furthermore, prior numerical and experimental investigations have revealed that, for small values of signal power, the displacement amplitude depends linearly on the applied voltage ${ }^{14,15}$. Consequently, the streaming is expected to scale quadratically with the applied voltage (i.e., streaming velocity $\propto \mathrm{V}_{\mathrm{PP}}{ }^{2}$ ). To investigate this scaling, we employed particle image velocimetry to measure the average velocities normal to the ciliary bands at sites indicated by magenta boxes in Fig. $\mathbf{3 a}$ and $\mathbf{3 b}$. Fig. $\mathbf{3 c}$ and $\mathbf{3 d}$ show that this quadratic relation is reasonably well satisfied by the ciliary bands. We note that the vertical streaming velocity of the + ciliary band is larger compared to the - configuration; likely due to the closer placement of the innermost cilia tips of the + ciliary band, which leads to each half band contributing more to the vertical streaming.

We reproduced the streaming flow patterns via numerical simulations based on the established perturbation approach ${ }^{14,16}$. Briefly, the perturbation approach expresses the fluid response to acoustic actuation as a sum of the first-order harmonic fields $\left(\mathbf{v}_{1}, \mathrm{p}_{1}, \rho_{1}\right)$ and the second-order steady fields $\left(\mathbf{v}_{2}, \mathrm{p}_{2}, \rho_{2}\right)$, e.g., $\mathbf{v}=\varepsilon \mathbf{v}_{\mathbf{1}}+\varepsilon^{2} \mathbf{v}_{\mathbf{2}}+\cdots$, where $\varepsilon$ is an appropriate smallness parameter ${ }^{16}$. This approach represents the steady flow field induced by the ciliary bands of the microrobot in a fluid by a second-order system of equations $\left(\mathbf{v}_{2}, \mathrm{p}_{2}, \rho_{2}\right)$, which, in turn, is driven by the body force and mass source terms stemming from nonlinear interactions of the time-harmonic first-order fields $\left(\mathbf{v}_{1}, \mathrm{p}_{1}, \rho_{1}\right)$. Further details of the theoretical and numerical formulation can be found in Supplementary text. The numerical simulations yielded the same 
qualitative flow patterns (Fig. $\mathbf{3 e}$ and $\mathbf{3 f}$ ) as the experimental results and therefore can serve as a useful tool in assessing the flow patterns for different configurations of ciliary arrays.

Engineering ciliary bands did not show any evidence of resonance characteristics. Nonetheless, we observed frequency dependent behaviour of our system. Specifically, we observed that the performance of the system, with regards to generation of streaming, was best at $68.5 \mathrm{kHz}$. This can be attributed to the fact that the piezo transducer's oscillation amplitude coupled with glass slide is maximum at this frequency, which is likely to be one of the resonances of the piezo/glass system, see also supporting Fig. S6. Our theoretical analysis reveals that the relative strength of the forcing terms with respect to the viscous terms in the second-order equations is determined by the frequency Reynolds number, $\operatorname{Re}_{f}=2 \pi f \Delta \mathrm{sL} / \nu$, where $\Delta \mathrm{s}$ is the oscillation amplitude, and the separation of time and length scales between the acoustic actuation and streaming flow (see also eq. 4). Therefore, these ciliary bands can generate motion as long as their oscillation frequency is sufficiently high to introduce inertia in the second-order equations.

\section{Bioinspired Microrobot}

Microrobots could facilitate specialized tasks in medicine ${ }^{17}$, including surgical procedures and drug delivery to hard-to-access sites in vivo. Chemical ${ }^{18-26}$ and external field-driven, such as magnetic $^{27-35}$, light $^{36-38}$, electrical ${ }^{39}$, biohybrid ${ }^{40-42}$ or ultrasound ${ }^{43-48}$ - microrobots are attractive because they do not require an onboard power supply or intricate moving parts and allow wireless control of the microrobot. Nonlinear ultrasound provides an alternate and attractive method to generate propulsion in vivo. In this section, we analyse the swimming motion of our bioinspired microrobot. The applied ultrasound has a wavelength on the order of a centimetre $(\sim 22 \mathrm{~cm})$, which is at least two orders of magnitude larger than the microrobot $(\sim 280 \mu \mathrm{m})$. As a result, the swimmer is subjected to uniform pressure on all sides. This expectation is further corroborated by a control experiment performed on a swimmer lacking ciliary bands and under acoustic actuation of different frequencies and amplitudes (Fig. S7); the lack of resultant motion of the device suggests (i) a nearly-uniform acoustic pressure field, with no significant net force on the swimmer, and (ii) the radiation force of the acoustic wave from the piezo transducer does not contribute to any motion.

We designed + and - ciliary bands on the left and right sides, respectively, of the soft robot, as shown in the schematic in Fig. 4a. As the microrobot is released and exposed to ultrasound of $68.8 \mathrm{kHz}$ at $20 \mathrm{~V}_{\mathrm{PP}}$, it exhibited a left-to-right translational propulsion (along the direction of short axis). Fig. $\mathbf{4 b}$ demonstrates an image sequence depicting the microrobot travelling at $\sim 2.6 \mathrm{~mm} / \mathrm{s}$ (10 body lengths per second; Movie S4). The velocity of the microrobot should also scale quadratically with the voltage applied since the propulsion is predominately 
streaming driven. Similar to ultrasound ciliary bands, the microrobotic design did not exhibit any resonance characteristics. As long as the amplitude of the acoustic field was sufficiently high at a given voltage, the microrobot should propel. The trajectory of the microrobot, i.e. translating left-to-right (along the direction of short axis), is further dictated by the configuration of ciliary bands. Fig. 4c and $\mathbf{4 d}$ show the complex fluid flow profile produced by the microrobot, comprised of a series of CW and CCW vortices. As expected, the arrangement of those vortices is symmetrical across the short axis $\left(x x^{\prime}\right)$, of the swimmer. Consequently, the forces exerted by the fluid on the swimmer along the long axis $\left(y y^{\prime}\right)$ was balanced, resulting in a net forcing and concomitant propulsion solely along the short axis (see also Movie S5).

The Reynolds number of the bioinspired microrobot can be estimated as $\operatorname{Re}=u D / v \approx$ 0.7 , where $\mathrm{u}=2.6 \mathrm{~mm} / \mathrm{s}$ is the swimming velocity and $\mathrm{D}=280 \mu \mathrm{m}$ is the width of the microrobot. This value also suggests that the system is viscous-dominated and thus must not exhibit any noticeable motion, per the scallop theorem. However, since ultrasound causes the ciliary band to oscillate, we must consider the Reynolds number associated with the oscillation of the cilia. For ultrasound actuated systems, the steady flow field is driven by the nonlinear interactions of the harmonic first-order response of the fluid. This can also be deduced from the perturbation expansion approach where the second-order system of equations is driven by time-averaged nonlinear first-order terms that scale with $\mathrm{Re}_{f}$ (see also Supplementary text). Depending on $\mathrm{Re}_{f}$, these first-order response dependent forcing terms (second and third term on the left-hand side of Eq. S7 in Supplementary text) can introduce sufficient inertia in the system to achieve swimming, even for reciprocal motion, rendering the Scallop theorem inapplicable.

The first-order fluid response is represented by the linearized Navier-Stokes equations, Eq. S4 and S5) and can be expressed in terms of the non-dimensional frequency Reynolds number. We begin by defining the following non-dimensional quantities:

$$
\left.\hat{\rho}=\frac{\rho_{0}}{\widetilde{\rho}}, \quad \hat{\mathbf{v}}_{\mathbf{1}}=\frac{\mathbf{v}_{\mathbf{1}}}{\widetilde{\mathrm{v}}}, \quad \hat{\mathrm{t}}=\frac{\mathrm{t}}{\tilde{\tilde{\mathrm{t}}}}, \quad \hat{\mu}=\frac{\mu}{\widetilde{\mu}^{\prime}}, \quad \text { (eq. } 1\right)
$$

where $\tilde{\rho}, \tilde{v}, \tilde{t}$ and $\tilde{\mu}$ denote the characteristic scales for the first order velocity, density, time, and viscosity, respectively. Using these non-dimensional quantities, the first-order momentum equation can be expressed as

$$
\left.\operatorname{Re}_{f} \hat{\rho} \frac{\partial \hat{\mathbf{v}}_{\mathbf{1}}}{\partial \hat{\mathrm{t}}}=-\widehat{\nabla} \hat{\mathrm{p}}_{1}+\hat{\mu} \widehat{\nabla}^{2} \hat{\mathbf{v}}_{\mathbf{1}}+\left(\hat{\mu}_{\mathrm{b}}+\frac{1}{3} \hat{\mu}\right) \widehat{\nabla}\left(\widehat{\nabla} \cdot \hat{\mathbf{v}}_{\mathbf{1}}\right), \quad \text { (eq. } 2\right)
$$

where, as expected, the relative strength of the inertial and viscous terms at the first order is characterized by the frequency Reynolds number given as $\operatorname{Re}_{f}=\frac{\widetilde{\rho} \widetilde{\mathrm{V}}}{\widetilde{\mu}}$, with $\tilde{\mathrm{L}}$ being the characteristic length scale for the first-order system. Noting that the first-order (actuation) 
velocity scale can be expressed as $\tilde{v}=\omega \Delta s=2 \pi f \Delta s$ and denoting kinematic viscosity as $v=\frac{\widetilde{\mu}}{\widetilde{\rho}}$, the frequency Reynolds number can be expressed as $\operatorname{Re}_{f}=2 \pi f \Delta s \mathrm{~L} / \nu$.

Next, we consider the relative strength of the forcing term in the second-order momentum equation (eq. 3 ) with respect to the viscous terms.

$$
\rho_{0}\left\langle\frac{\partial \mathbf{v}_{2}}{\partial \mathrm{t}}\right\rangle+\left\langle\rho_{1} \frac{\partial \mathbf{v}_{1}}{\partial \mathrm{t}}\right\rangle+\rho_{0}\left\langle\left(\mathbf{v}_{\mathbf{1}} \cdot \nabla\right) \mathbf{v}_{\mathbf{1}}\right\rangle=-\nabla \mathrm{p}_{2}+\mu \nabla^{2} \mathbf{v}_{\mathbf{2}}+\left(\mu_{\mathrm{b}}+\frac{1}{3} \mu\right) \nabla\left(\nabla \cdot \mathbf{v}_{\mathbf{2}}\right)
$$

Specifically, the third term on the left-hand side of eq. 3 scales as $\rho_{0}\left\langle\left(\mathbf{v}_{\mathbf{1}} \cdot \nabla\right) \mathbf{v}_{\mathbf{1}}\right\rangle \sim \frac{\widetilde{\rho} \widetilde{v}^{2}}{\widetilde{L}}$, while the viscous term in eq. 3 scales as $\mu \nabla^{2} \mathbf{v}_{2} \sim \frac{\widetilde{\mu}_{s}}{\widetilde{\mathrm{L}}_{s}^{2}}$. Therefore, the relative strength of the forcing term with respect to the viscous terms is given by the ratio

$$
\frac{\widetilde{\rho} \widetilde{V}^{2}}{\widetilde{L}} \cdot \frac{\widetilde{L}_{s}^{2}}{\widetilde{\mu} \widetilde{V}_{s}} \sim \operatorname{Re}_{f} \frac{\widetilde{v}}{\widetilde{V}_{s}}\left(\frac{\widetilde{L}_{s}}{\widetilde{L}}\right)^{2} \sim \operatorname{Re}_{f} \frac{\widetilde{V}_{s}}{\widetilde{v}}\left(\frac{\tilde{t}_{s}}{\tilde{\mathfrak{t}}}\right)^{2} \quad \text { (eq. 4) }
$$

where $\tilde{v}_{S}, \tilde{L}_{s}$, and $\tilde{t}_{s}$ denote the characteristic scales for the streaming velocity, length, and time, respectively. Thus, the forcing term in the second-order system depends on the frequency Reynolds number as well as the separation of time and length (or velocity) scales between the first-order and the streaming flow. Consequently, a sufficiently large Reynolds number and adequately separated time and length scales can introduce significant inertia in the secondorder equations and therefore violate the contingency of the scallop theorem, to achieve propulsion even with a reciprocal actuation. Furthermore, since $\mathrm{Re}_{f}$ scales linearly with the frequency and amplitude of a vibrating object, the frequency Reynolds number of the ciliary bands can be engineered to have intermediate values, which implies that inertia can be introduced to produce propulsion at microscale even for reciprocal motion. The frequency Reynolds number of our oscillating cilium can be estimated as $\operatorname{Re}_{f}=2 \pi f \Delta \mathrm{sL} / \nu \approx 31.4-62.8$, where $f=100 \mathrm{kHz}$ is the excitation frequency and $\Delta s \approx 0.5-1.0 \mu \mathrm{m}$ (see also Fig. S4 and Movie S6).

The total time-averaged acoustic propulsive force $\mathbf{F}_{\mathbf{A}}$, experienced by the microrobot can be expressed as

$$
\mathbf{F}_{\mathbf{A}}=\oint_{\partial \Omega_{1}}\left\langle\boldsymbol{\sigma}_{2}\right\rangle \cdot \mathbf{n d A}+\oint_{\partial \Omega_{1}} \rho_{0}\left\langle\mathbf{v}_{\mathbf{1}} \mathbf{v}_{\mathbf{1}}\right\rangle \cdot \mathbf{n d A} \quad \text { (eq. 5) }
$$

where $\partial \Omega_{1}$ represents the surface of the microrobot. Here, the first term accounts for the contribution from the stress $\left\langle\sigma_{2}\right\rangle$ developed by localized acoustic microstreaming, while the second term represents the nonlinear interactions of the first-order harmonic velocity response. As revealed by our scaling analysis, both the first-order response as well as the forcing terms for the second-order equations depend on $\mathrm{Re}_{f}$. Therefore, each of the terms on the right-hand side of Eq. 5 , can be tuned by modifying $\mathrm{Re}_{f}$. Consequently, propulsion can be achieved by 
tuning $\mathrm{Re}_{f}$. Therefore, the starfish larva-inspired microrobot circumvents the scallop theorem by introducing high-frequency oscillation of ciliary bands which induces inertial forces and subsequent propulsion.

\section{Microparticle Transport}

Next, we introduce a microparticle trapping strategy inspired by the feeding mechanism of a starfish larvae ${ }^{1}$. Briefly, this mechanism is characterized by the juxtaposition of ciliary arrays that beat in reverse, generating a specific flow field that facilitates the transport of particles and nutrients to the larva's surface for subsequent capture. Correspondingly, we design an analogous structure that incorporates a combinatorial arrangement of + and - ciliary bands, as shown in Fig. 5a.

When a synthetic band with this arrangement is exposed to ultrasound, microparticles in close vicinity of the + ciliary band are initially attracted to and travelled along the left ciliary array, then at the centre of the + ciliary band are pushed away at velocities of up to $2 \mathrm{~mm} / \mathrm{s}$ (Movie S7). Were this a stand-alone + ciliary band, the trajectories of these particles would have followed the symmetrical vortices, as in Fig. 3a. However, the presence of an adjacent ciliary band breaks the symmetry of a stand-alone + ciliary band and instead pulls these particles towards the right half of the + ciliary band. Subsequently, in accordance with the source-like flow profile generated by a + ciliary band, these particles move away from the + ciliary band. Given that the strength of the source diminishes with distance from the + ciliary band, the effect of the adjacent sink becomes more prominent as the microparticles progress, causing them to be transported towards the - ciliary band, as shown in Fig. $\mathbf{5 b}$. Fig. $\mathbf{5 c}$ and $\mathbf{5 d}$ demonstrate the trajectory and velocity behaviour of microparticles exposed to the trapping flow field provoked by a trapping ciliary band configuration. As expected, the particle decelerates as it moves away from the + band, then accelerates upon approach towards the - band. Overall, this arrangement of adjacent + and - ciliary bands allow the migration of microparticles from one ciliary band to another. Combined with an efficient capture strategy that further siphons these particles into the body of the synthetic structure, this transport mechanism can be used to design efficient microrobotic systems that can attract and capture particles of interest from the surrounding flow field.

\section{Discussion}

We developed ultrasound-activated synthetic ciliary bands inspired by the natural ciliary arrangements on the surface of starfish larva. When our planar ciliary bands are angled toward each other (+), fluid is pushed away from the surface on which the cilia are arranged. In contrast, when the cilia are angled away from each other $(-)$, liquid is forced in toward the bands. Thus, we have developed an ultrasound-actuated ciliary arrangement mimicking a source and sink in 
an artificial system. We further incorporated these aspects to develop a new physical and design principle for acoustic-based microrobots. Our starfish larva-inspired microrobot overcomes the scallop theorem due to its ciliary bands, as their high-frequency oscillations induce inertial forces and consequent propulsion, rendering the contingency of the theorem invalid. Finally, by placing + and - ciliary bands adjacent to each other, we demonstrate a microparticle transport mechanism analogous to a starfish larva's feeding mechanism.

Since microorganisms live in an environment where inertial effects are negligible, their cilia must produce nonlinear whip-like motion, i.e., an asymmetric beat pattern is executed to overcome the reversibility of low-Re flows, as required by the scallop theorem. The ultrasound cilia and ciliary bands are unique in comparison to natures' cilia. Specifically, unlike natural cilia, ultrasound cilia undergo reciprocal motion to produce a flow. This apparent contradiction with the scallop theorem can be attributed to the oscillation frequency of ultrasound cilia that is at least three orders of magnitude higher than its natural counterpart. As revealed by our theoretical analysis, it is this separation of time scales between the (second-order) fluid response and the (firs-order) acoustic actuation that is responsible for the bulk fluid motion, despite the reciprocal motion of cilia. Interestingly, when synthetic cilia were exposed to lowfrequency ultrasound between 1 to $100 \mathrm{~Hz}$ (see also Fig. S4), individual particles demonstrate to and fro motion without any net displacement, further confirming the crucial role of excitation frequency.

We believe the present work introduces a new design space for externally-actuated fielddriven microrobots and the engineering of cilia and ciliary bands that are not exclusive to ultrasound-based systems. Engineering cilia that could produce reciprocal motion in order to introduce inertia are much simpler in terms of their fabrication and operation. The concept can be transferred to non-ultrasound methods, for example in magnetic field-driven systems. Recent studies on magnetism-based synthetic cilia mimic the nonreciprocal whip-like beating pattern of biological cilia; however, engineering these devices requires multi-step fabrication, and they are challenging to scale down to the microscale. Achieving reciprocal motion in the $\mathrm{kHz}$ domain with magnetically-doped engineered cilia may introduce inertia at microscales but may be challenging due to (i) the need to rapidly switch the magnetic field and (ii) magnetic hysteresis, the retention of magnetic alignment after a field is removed. In addition to magnetic-field driven systems, light-driven liquid crystal-doped polymeric structures have been shown to generate fast movement and to enable rapid switching in the $\mathrm{kHz}$ domain, and thus could become a potential candidate mechanism for propulsion via reciprocal motion.

In our current experiments, depending on the fabrication of the ciliary bands and the corresponding oscillation patterns, we observed smaller counter-rotating vortices at the innermost cilia, in addition to the larger counter-rotating vortices above the ciliary bands (Fig. 
S8). Future work will aim to thoroughly investigate the oscillation patterns of the cilia, including the effect of different fabrication processes and material stiffness. We look forward to studying the performance of our ultrasound-based ciliary bands in terms of fluid flow, propulsion, and particle transport near walls and in confined channels in biologically-relevant fluid mediums under physiologically-relevant conditions, including non-pulsatile and pulsatile flow. An immediate next step also involves investigating their performance in non-Newtonian liquids such as blood, viscoelastic mediums, and shear-thinning gels. Currently, the angle of our ciliary bands are fixed, we plan that a light-activated liquid crystal polymer can be used to dynamically change the orientations of ciliary bands, i.e. switch from + to - arrangements and vice versa, thereby enabling the development of a robotic system that closely mimics its natural counterparts in propulsion and feeding (trapping) mechanisms. Finally, the concept of + and ciliary bands can be utilized in lab-on-a-chip systems to realize label-free trapping, fluid mixing and pumping at low Reynolds fluid low, and separation of particles for portable diagnostics applications. 


\section{Materials and Methods}

Fabrication Method. Ciliary bands were fabricated using a custom-built projection UV photolithography method developed on an inverted microscope (Fig. S1). A UV lamp (NIKON, Intensilight C-HGFI) was mounted onto an inverted epi-fluorescence microscope (NIKON, Eclipse Ti) and used to irradiate a high-resolution photomask (CAD/Art Services, Inc.) inserted into the field stop of the microscope. UV light passed through the mask, became focused through a 20x objective, and polymerized the photosensitive mixture (see Materials) downsized by a factor of $\sim 16.3$. In exposed regions, the negative photoresist polymerized according to the photomask pattern. The UV exposure time was controlled by an electric shutter system (Vincent Associates, VCM-D1) to fully polymerize the ciliary band and varied between $500-3000 \mathrm{~ms}$ at different UV-intensity levels $(12.5$ - 100.0\%) selected on the UV lamp.

Materials. The photosensitive polymer mixture used in this project consists of photocrosslinkable polyethylene glycols (PEGs) and a photo-initiator. The ratios of each component in the mixture were: 50\% (v/v) PEG diacrylate with a molecular weight of 700 (PEG 700, SigmaAldrich), 30\% (v/v) PEG diacrylate with a molecular weight of 258 (PEG 258, Sigma-Aldrich), 15\% (v/v) TE buffer (100 TE, Sigma-Aldrich), and 5\% (v/v) photo-initiator 2-hydroxy-2-methyl1-phenyl-propan-1-one (Darocur 1173, Sigma-Aldrich) as photo-initiator. In addition, a droplet $(\sim 50 \mu \mathrm{l})$ each of fluorescent Rhodamine B (Sigma-Aldrich) and food dye were added to facilitate the focusing of the photomask and to achieve better contrast for the microrobots suspended in DI water.

Acoustic Setup. The acoustic system (Fig. S3) was built on a $25 \mathrm{~mm}$ x $75 \mathrm{~mm}$ x $1 \mathrm{~mm}$ glass slide (Menzel) whereon a transducer disc (Murata, 7BB-27-4L0) was attached with epoxy resin (2-K-Epoxidkleber, UHU Schnellfest). Once the ciliary band and other microarchitectures were fabricated and cleaned (IPA, Sigma-Aldrich) on the acoustic device, they were transferred onto the microscope stage. A solution consisting of $10: 1$ by volume DI water and tracer $5.7 \mu \mathrm{m}$ particles (Polysciences) was placed on top of the microstructures. A confined "liquid manipulation chamber" was developed by applying a coverslip (22 $\mathrm{mm}$ x $22 \mathrm{~mm}$ ) to the droplet $(\sim 150 \mu \mathrm{l})$ containing microstructures and tracer particles. The piezo transducer was then connected to the function generator (AFG 3011C, Tektronix) via an amplifier (Thurlby Thandar Instruments, WA301) to generate acoustic fields with adjustable frequencies and voltages within the liquid. The whole setup was mounted on an inverted microscope.

Imaging and Data Analysis. Experiments were performed on a Zeiss Axiovert 200M inverted microscope equipped with a fluorescent camera (Coolsnap EZ, Photometrics) on its side port and a high-speed camera (CHRONOS 1.4, Kron Technologies) on its top port. Recoded videos were processed using ImageJ and further analysed using a Particle Image Velocimetry (PIV) 
approach ${ }^{49,50}$. Custom-built MATLAB scripts, PIV processing parameters, and raw data sets are available upon request from the corresponding author.

Numerical Simulation. The numerical simulations reported in this work were conducted using the commercial COMSOL Multiphysics finite element software. For the first-order system of equations, which is indicative of the acoustic response of the system, we sought time-harmonic solutions with frequency equal to the actuation frequency. In contrast, we sought steady solutions for the second-order components, which represent the time-averaged response of the system. These systems of equations were solved in a sequential manner wherein the first-order solution was used to calculate the forcing terms in the second-order system of equations. We performed 2D simulations on a rectangular domain that was chosen to be sufficiently large to model quiescent fluid around the microswimmer. The oscillating cilia were assigned a non-zero Dirichlet boundary condition for the first-order velocity and a homogeneous Dirichlet boundary condition for the second-order Lagrangian velocity. We employed a triangular mesh with P1-P2 composite elements for the pressure and velocity, where P1 and P2 denote triangular elements with Lagrange polynomials of order 1 and 2 respectively. For both the first- and second-order system of equations, a direct solver was utilized to obtain the solution. 
Acknowledgments. This project has received funding from the European Research Council (ERC) under the European Union's Horizon 2020 research and innovation programme grant agreement No 853309 (SONOBOTS) and ETH Research Grant ETH-08 20-1. We thank Prof. Juerg Dual and Mr. Alen Pavlic for assisting us with the frequency characterization of the Piezo transducer.

Author Contributions. D.A. initiated and supervised the project. C.D. performed all the experiments and performed data analysis with feedback from N.N. and D.A. N.N. contributed to the theoretical understanding and developed the numerical simulation. All authors contributed to the experimental design, scientific presentation, discussion, and wrote the manuscript.

Competing Interest Statement. The authors declare no competing financial interests. 


\section{References}

1. Gilpin, W., Prakash, V. N. \& Prakash, M. Vortex arrays and ciliary tangles underlie the feeding-swimming trade-off in starfish larvae. Nat. Phys. 13, 380-386 (2017).

2. Evans, B. A. et al. Magnetically actuated nanorod arrays as biomimetic cilia. Nano Lett. 7, 1428-1434 (2007).

3. Vilfan, M. et al. Self-assembled artificial cilia. Proc. Natl. Acad. Sci. 107, 1844-1847 (2010).

4. Shields, A. R. et al. Biomimetic cilia arrays generate simultaneous pumping and mixing regimes. Proc. Natl. Acad. Sci. 107, 15670-15675 (2010).

5. Gu, H. et al. Magnetic cilia carpets with programmable metachronal waves. Nat. Commun. 11, 1-10 (2020).

6. Dong, X. et al. Bioinspired cilia arrays with programmable nonreciprocal motion and metachronal coordination. Sci. Adv. 6, eabc9323 (2020).

7. Van Oosten, C. L., Bastiaansen, C. W. M. \& Broer, D. J. Printed artificial cilia from liquidcrystal network actuators modularly driven by light. Nat. Mater. (2009) doi:10.1038/nmat2487.

8. Milana, E., Gorissen, B., Peerlinck, S., De Volder, M. \& Reynaerts, D. Artificial soft cilia with asymmetric beating patterns for biomimetic low-Reynolds-number fluid propulsion. Adv. Funct. Mater. 29, 1900462 (2019).

9. Orbay, S., Ozcelik, A., Bachman, H. \& Huang, T. J. Acoustic actuation of in situ fabricated artificial cilia. J. Micromechanics Microengineering 28, 25012 (2018).

10. Qiu, T. et al. Swimming by reciprocal motion at low Reynolds number. Nat. Commun. 5, 1-8 (2014).

11. Trouilloud, R., Tony, S. Y., Hosoi, A. E. \& Lauga, E. Soft swimming: Exploiting deformable interfaces for low Reynolds number locomotion. Phys. Rev. Lett. 101, 48102 (2008).

12. Purcell, E. M. Life at low Reynolds number. Am. J. Phys. (1977) doi:10.1119/1.10903.

13. Schlichting, H., Gersten, K., Schlichting, H. \& Gersten, K. Fundamentals of BoundaryLayer Theory. in Boundary-Layer Theory (2017). doi:10.1007/978-3-662-52919-5_2.

14. Nama, N., Huang, P. H., Huang, T. J. \& Costanzo, F. Investigation of acoustic streaming patterns around oscillating sharp edges. Lab Chip (2014) doi:10.1039/c4lc00191e.

15. Huang, P.-H. et al. An acoustofluidic micromixer based on oscillating sidewall sharpedges. Lab Chip 13, 3847-3852 (2013).

16. Sadhal, S. S., Laurell, T. \& Lenshof, A. Analysis of acoustic streaming by perturbation methods. in Microscale Acoustofluidics 256-311 (Royal Society of Chemistry London, 2014).

17. Schmidt, C. K., Medina-Sánchez, M., Edmondson, R. J. \& Schmidt, O. G. Engineering microrobots for targeted cancer therapies from a medical perspective. Nat. Commun. 11, 1-18 (2020).

18. Dekanovsky, L. et al. Chemically programmable microrobots weaving a web from hormones. Nat. Mach. Intell. 2, 711-718 (2020).

19. Hermanová, S. \& Pumera, M. Biocatalytic Micro-and Nanomotors. Chem. Eur. J. 26, 11085-11092 (2020).

20. Simmchen, J. et al. Topographical pathways guide chemical microswimmers. Nat. Commun. 7, 10598 (2016).

21. Ma, X. et al. Enzyme-powered hollow mesoporous Janus nanomotors. Nano Lett. 15, 7043-7050 (2015).

22. Mei, Y., Solovev, A. A., Sanchez, S. \& Schmidt, O. G. Rolled-up nanotech on polymers: from basic perception to self-propelled catalytic microengines. Chem. Soc. Rev. 40, 2109-2119 (2011).

23. Sánchez, S., Soler, L. \& Katuri, J. Chemically powered micro-and nanomotors. Angew. Chemie Int. Ed. 54, 1414-1444 (2015).

24. Hortelão, A. C. et al. LipoBots: Using Liposomal Vesicles as Protective Shell of UreaseBased Nanomotors. Adv. Funct. Mater. 30, 2002767 (2020).

25. Somasundar, A. et al. Positive and negative chemotaxis of enzyme-coated liposome motors. Nat. Nanotechnol. 14, 1129-1134 (2019). 
26. Das, S. et al. Harnessing catalytic pumps for directional delivery of microparticles in microchambers. Nat. Commun. 8, 1-10 (2017).

27. Hu, W., Lum, G. Z., Mastrangeli, M. \& Sitti, M. Small-scale soft-bodied robot with multimodal locomotion. Nature 554, 81-85 (2018).

28. Fan, X., Dong, X., Karacakol, A. C., Xie, H. \& Sitti, M. Reconfigurable multifunctional ferrofluid droplet robots. Proc. Natl. Acad. Sci. 117, 27916-27926 (2020).

29. Tasci, T. O., Herson, P. S., Neeves, K. B. \& Marr, D. W. M. Surface-enabled propulsion and control of colloidal microwheels. Nat. Commun. 7, 1-6 (2016).

30. Yang, T. et al. Microwheels on microroads: Enhanced translation on topographic surfaces. Sci. Robot. 4, (2019).

31. Zhang, L. et al. Artificial bacterial flagella: Fabrication and magnetic control. Appl. Phys. Lett. 94, 64107 (2009).

32. Yan, X. et al. Multifunctional biohybrid magnetite microrobots for imaging-guided therapy. Sci. Robot. 2, (2017).

33. lacovacci, V. et al. Retrieval of magnetic medical microrobots from the bloodstream. in 2019 International Conference on Robotics and Automation (ICRA) 2495-2501 (2019).

34. Cui, J. et al. Nanomagnetic encoding of shape-morphing micromachines. Nature 575, 164-168 (2019).

35. Alcântara, C. C. J. et al. Mechanically interlocked 3D multi-material micromachines. Nat. Commun. 11, 1-8 (2020).

36. Palagi, S. et al. Structured light enables biomimetic swimming and versatile locomotion of photoresponsive soft microrobots. Nat. Mater. 15, 647-653 (2016).

37. Shahsavan, $\mathrm{H}$. et al. Bioinspired underwater locomotion of light-driven liquid crystal gels. Proc. Natl. Acad. Sci. 117, 5125-5133 (2020).

38. Li, M., Wang, X., Dong, B. \& Sitti, M. In-air fast response and high speed jumping and rolling of a light-driven hydrogel actuator. Nat. Commun. 11, 1-10 (2020).

39. Alapan, Y., Yigit, B., Beker, O., Demirörs, A. F. \& Sitti, M. Shape-encoded dynamic assembly of mobile micromachines. Nat. Mater. 1 (2019).

40. Williams, B. J., Anand, S. V, Rajagopalan, J. \& Saif, M. T. A. A self-propelled biohybrid swimmer at low Reynolds number. Nat. Commun. 5, 1-8 (2014).

41. Li, Z. et al. Biohybrid valveless pump-bot powered by engineered skeletal muscle. Proc. Natl. Acad. Sci. 116, 1543-1548 (2019).

42. Magdanz, V. et al. IRONSperm: Sperm-templated soft magnetic microrobots. Sci. Adv. 6, eaba5855 (2020).

43. Zhou, Q., Sariola, V., Latifi, K. \& Liimatainen, V. Controlling the motion of multiple objects on a Chladni plate. Nat. Commun. 7, 1-10 (2016).

44. Ahmed, D. et al. Selectively manipulable acoustic-powered microswimmers. Sci. Rep. (2015) doi:10.1038/srep09744.

45. Ahmed, D. et al. Artificial swimmers propelled by acoustically activated flagella. Nano Lett. 16, 4968-4974 (2016).

46. Ahmed, D. et al. Neutrophil-inspired propulsion in a combined acoustic and magnetic field. Nat. Commun. 8, 1-8 (2017).

47. Ren, L. et al. 3D steerable, acoustically powered microswimmers for single-particle manipulation. Sci. Adv. 5, eaax3084 (2019).

48. Aghakhani, A., Yasa, O., Wrede, P. \& Sitti, M. Acoustically powered surface-slipping mobile microrobots. Proc. Natl. Acad. Sci. 117, 3469-3477 (2020).

49. Schneider, C. A., Rasband, W. S. \& Eliceiri, K. W. NIH Image to ImageJ: 25 years of image analysis. Nature Methods (2012) doi:10.1038/nmeth.2089.

50. Thielicke, W. \& Stamhuis, E. J. PIVlab - Towards User-friendly, Affordable and Accurate Digital Particle Image Velocimetry in MATLAB. J. Open Res. Softw. 2, (2014). 
Figures and Legends

a Starfish Larva

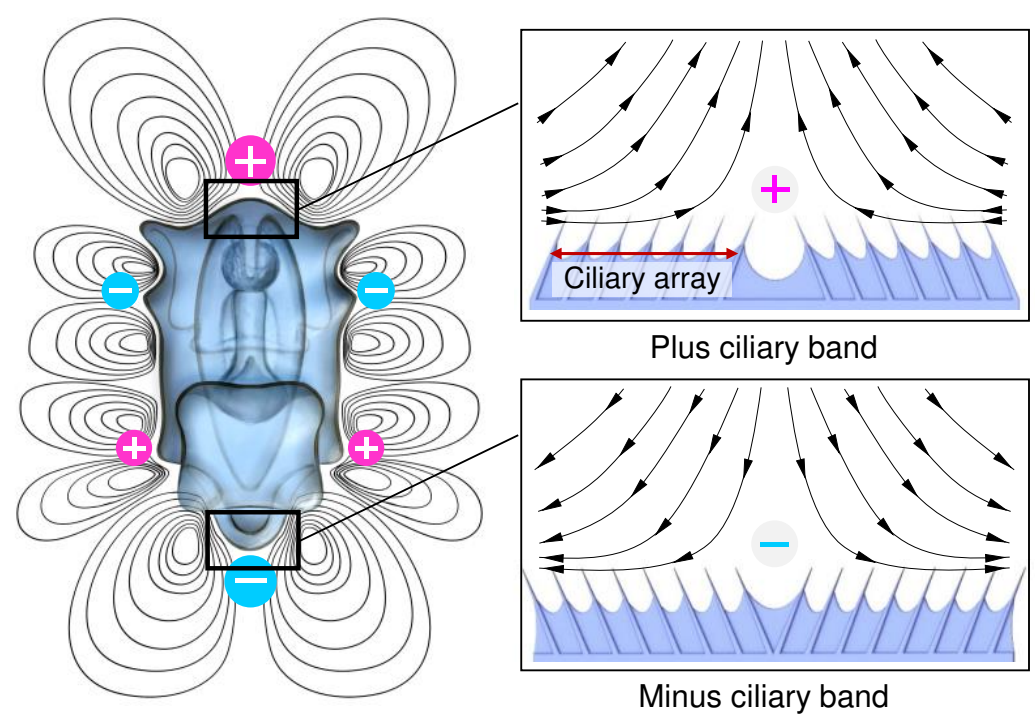

b Bioinspired Microrobot

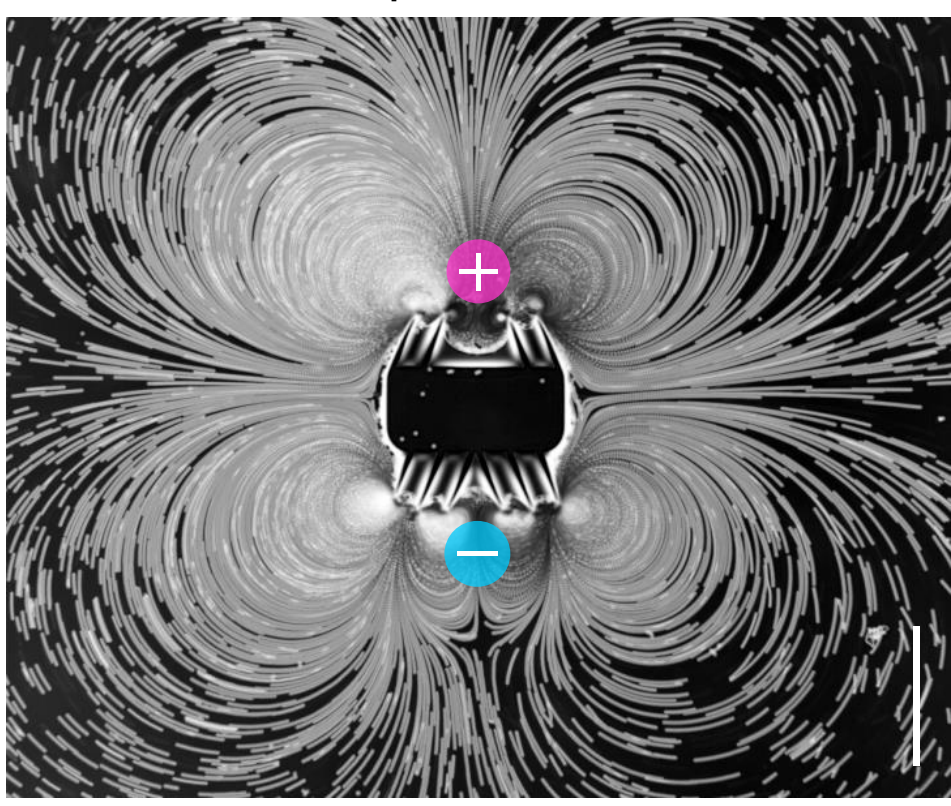

Fig. 1 | Starfish larva-inspired ultrasound ciliary band designs. a. A starfish larva exhibits a complex flow profile of counter-rotating vortices generated by series of + and - ciliary bands arranged on its body protuberances. Inset: (top) A + ciliary band comprises a pair of angled ciliary arrays that face each other and causes the liquid to flow away on a plane perpendicular to the centre of the ciliary band. (Bottom) A - ciliary band consists of two ciliary arrays oriented away from each other and directs the liquid flow to the centre of the band. b. A starfish-inspired microrobot consisting of $\mathrm{a}+$ (top) and $\mathrm{a}-$ (bottom) ciliary band placed in a tracer solution. The ciliary bands oscillate when actuated by ultrasound, producing complex flow profiles similar to those observed with its biological counterpart. Scale bar, $250 \mu \mathrm{m}$. 

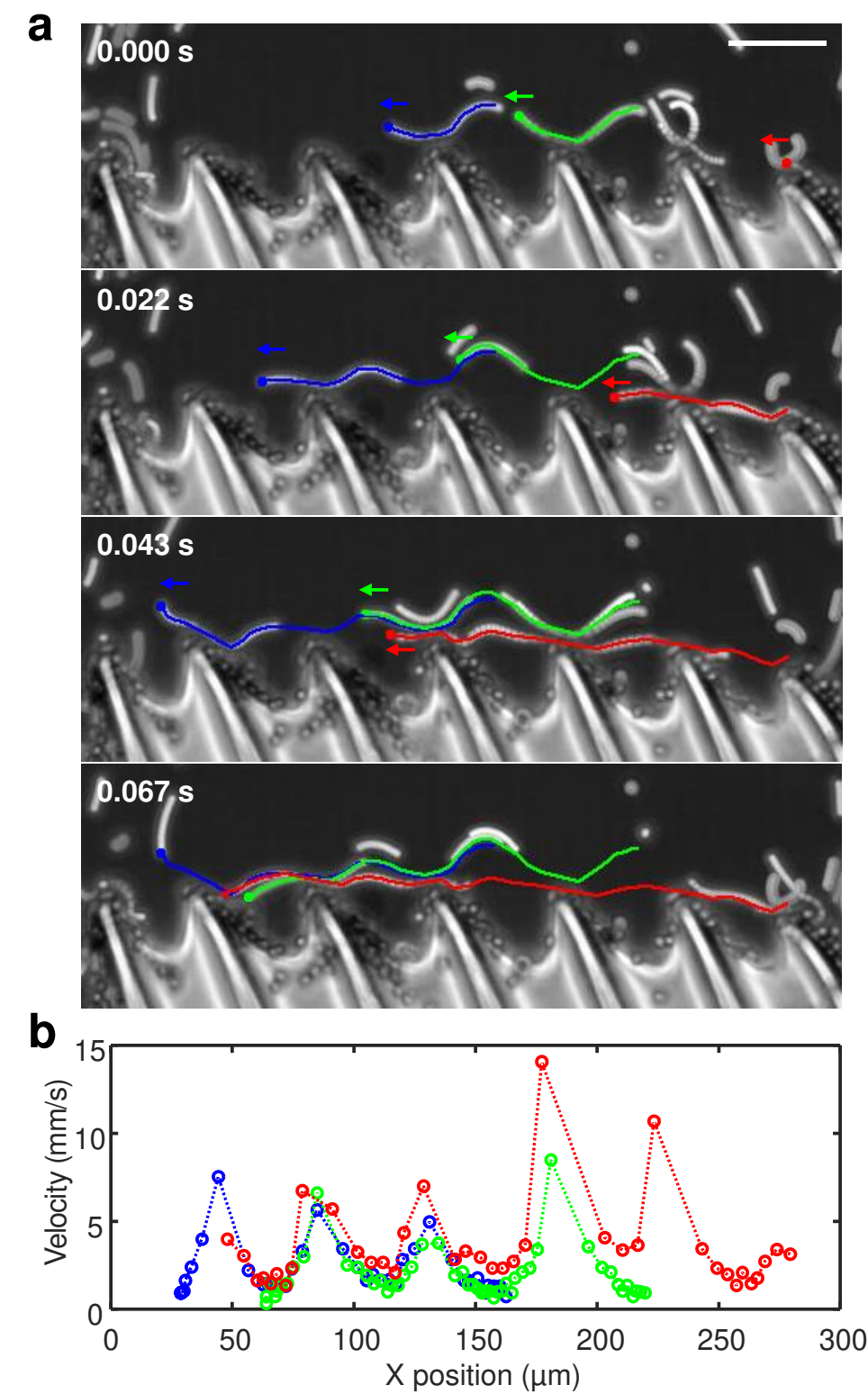

Fig. 2 | Tangential flow along angled ciliary array when exposed to ultrasound. a. Image sequences demonstrate $\sim 6 \mu \mathrm{m}$ tracer particles, indicated by red, green, and blue lines, travelling along one cilium tip to the next from right-to-left in the direction the tips are angled at excitation frequency and amplitude of $33.7 \mathrm{kHz}$ and $5 \mathrm{~V}_{\mathrm{PP}}$, respectively (see also Movie S1). b. Velocity analysis of the tracers revealed a cyclic acceleration/deceleration pattern. Particles reached maximum speeds when they approached a ciliary tip, followed by a deceleration phase. Scale bar, $50 \mu \mathrm{m}$. 
a

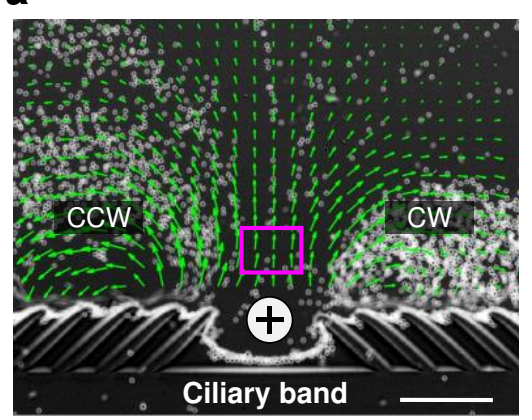

b

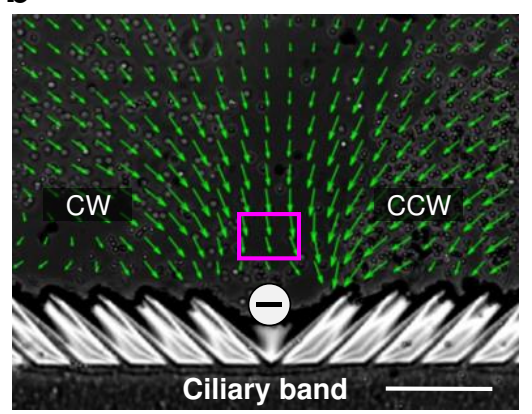

C

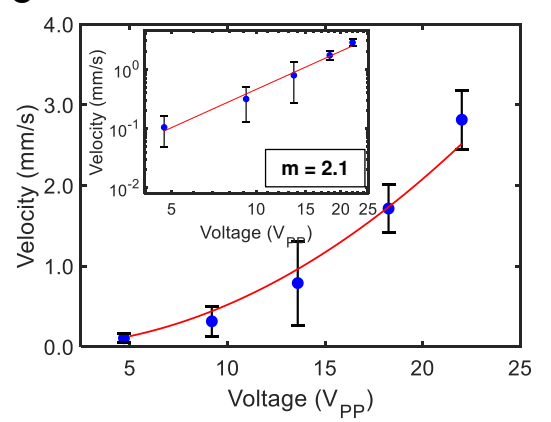

d

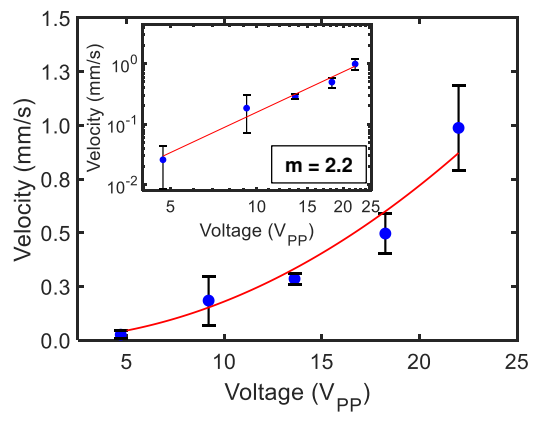

e

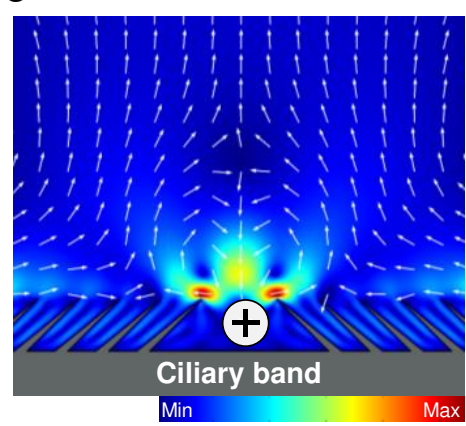

f

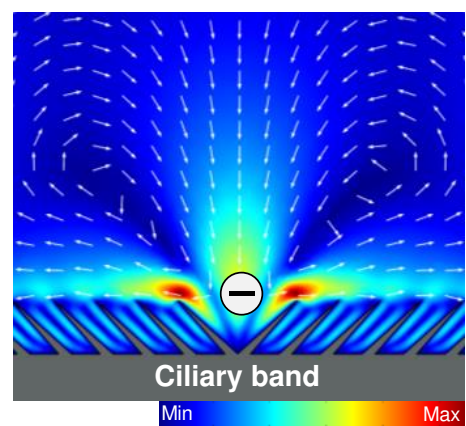

Fig. 3 | Experimental, characterization, and numerical demonstration of bioinspired ultrasound ciliary bands (Movie S2). a. PIV generated velocity fields of a + ciliary band that causes the liquid to flow away on a plane perpendicular to the centre of the ciliary band at excitation frequency and amplitude of $68.7 \mathrm{kHz}$ and $20 \mathrm{~V}_{\mathrm{PP}}$, respectively. b. PIV generated velocity fields of a - ciliary band, which directs the liquid flow to the centre of the band at $68.8 \mathrm{kHz}$ and $22 \mathrm{~V}_{\mathrm{PP}}$, respectively. Plots of average velocities of c. + and d. - ciliary bands at sites indicated by magenta boxes in $\mathbf{a}$. and $\mathbf{b}$. versus voltage applied. The particles immersed in water transport at a speed proportional to the square of the voltage applied and this quadratic relation is reasonably well satisfied as indicated by the log plots. In each data point, 400 velocity measurements are averaged and the standard deviation is calculated and they are represented as black error bars. Numerical simulation of microstreaming using perturbation approach of $\mathbf{e}$. + ciliary band and f. - ciliary band. Scale bar, $100 \mu \mathrm{m}$. 
a

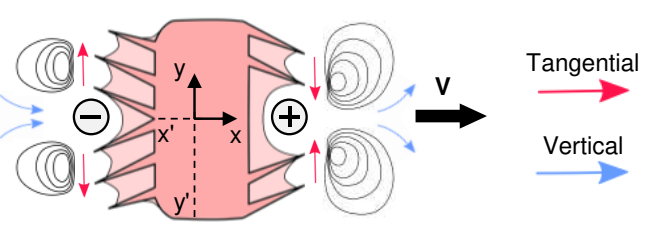

C

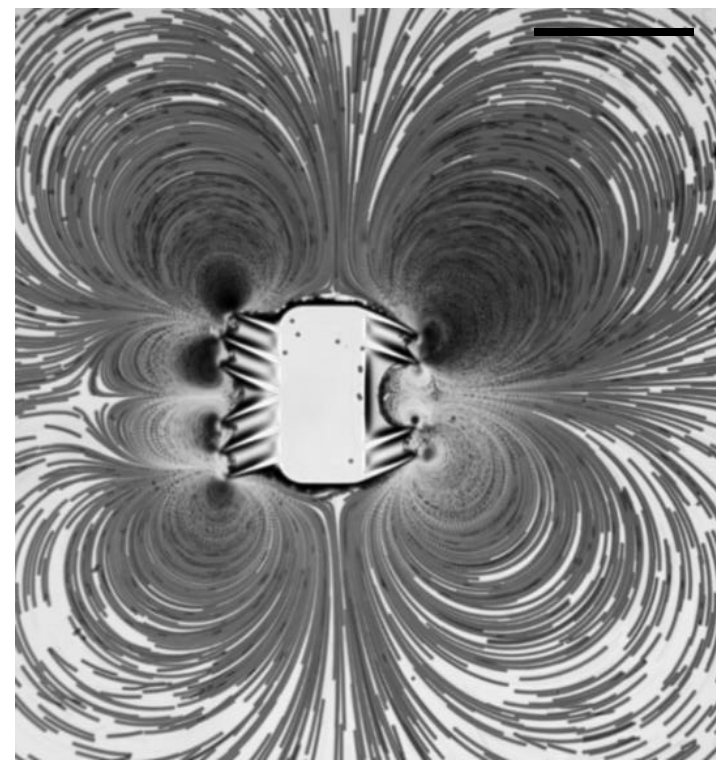

b

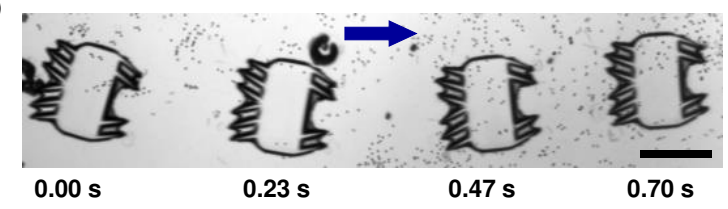

d

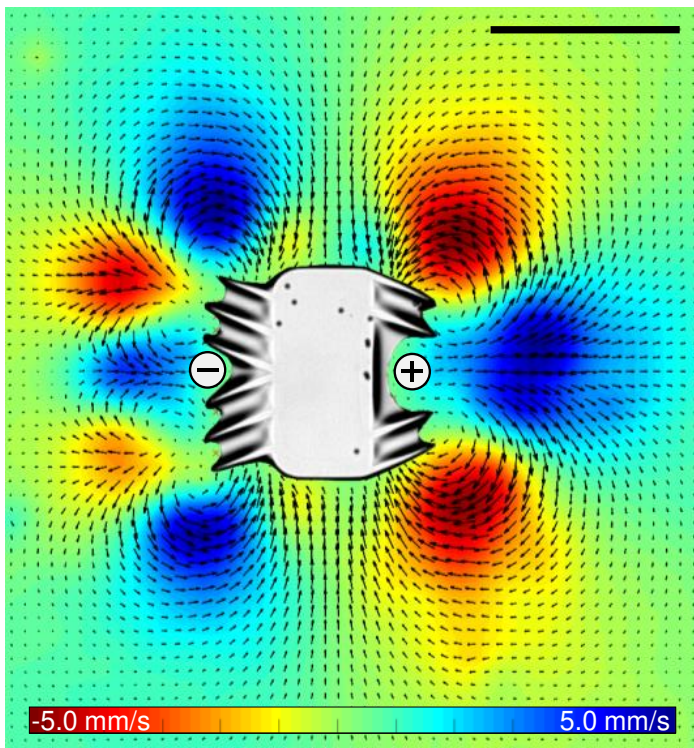

Fig. 4 | Propulsion of bioinspired ultrasound microrobot. a. Schematic of an artificial microrobot consisting of a - ciliary band on the left and a + ciliary band on the right. Red and blue arrows indicate tangential and vertical velocity, respectively. b. Superimposed time-lapse image of controlled translation motion of a microrobot at excitation frequency and amplitude of $68.8 \mathrm{kHz}$ and $20 \mathrm{~V}_{\mathrm{PP}}$, respectively (see also Movie S4). c. Image sequence illustrating the streaming flow profile of the bioinspired microrobot. A stack of 150 images was used; the video was captured using a high-speed camera at a framerate of $1069 \mathrm{fps}$ (see also Movie S5). d. PIV generated velocity fields demonstrate the complex flow behaviour developed in the surrounding liquid. Minimum and maximum flow velocities are marked by red and blue regions, respectively. Scale bars, $250 \mu \mathrm{m}$. 
a

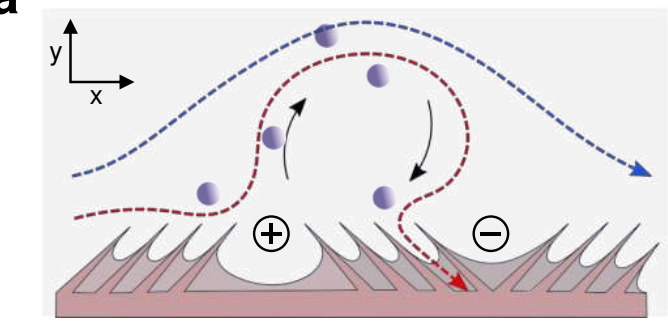

b

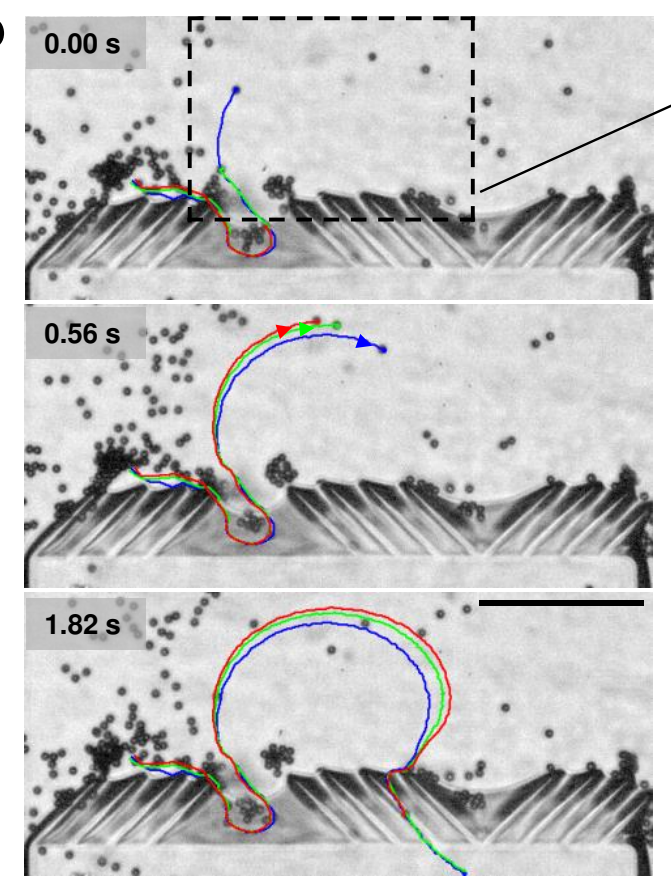

C
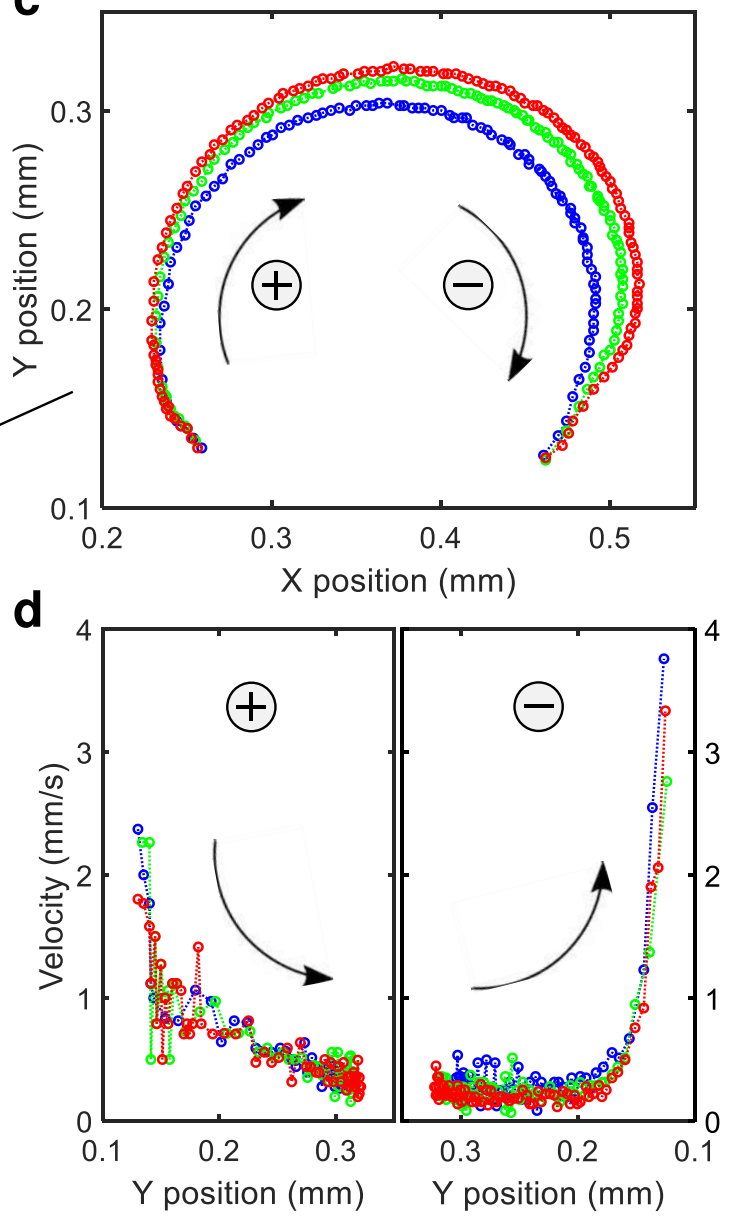

Fig. 5 | Bioinspired microparticle trapping using a combination of + and - ciliary bands. a. Schematic of a bioinspired trapping mechanism consisting of a + ciliary band adjacent to a ciliary band (see also Movie S7). b. Image sequence demonstrating microparticles becoming trapped in the - ciliary band, as indicated by red, green, and blue trajectories at $68.5 \mathrm{kHz}$ and $20 V_{\mathrm{PP}}$. c. Expanded spatial trajectories of microparticles that became trapped by the flow field produced from the adjacent + and - ciliary bands. $d$. Plots of microparticle velocity versus $y$ position: the left panel indicates the source and the right panel the sink behaviour of the trapping ciliary band. Scale bar, $200 \mu \mathrm{m}$. 


\section{Figures}
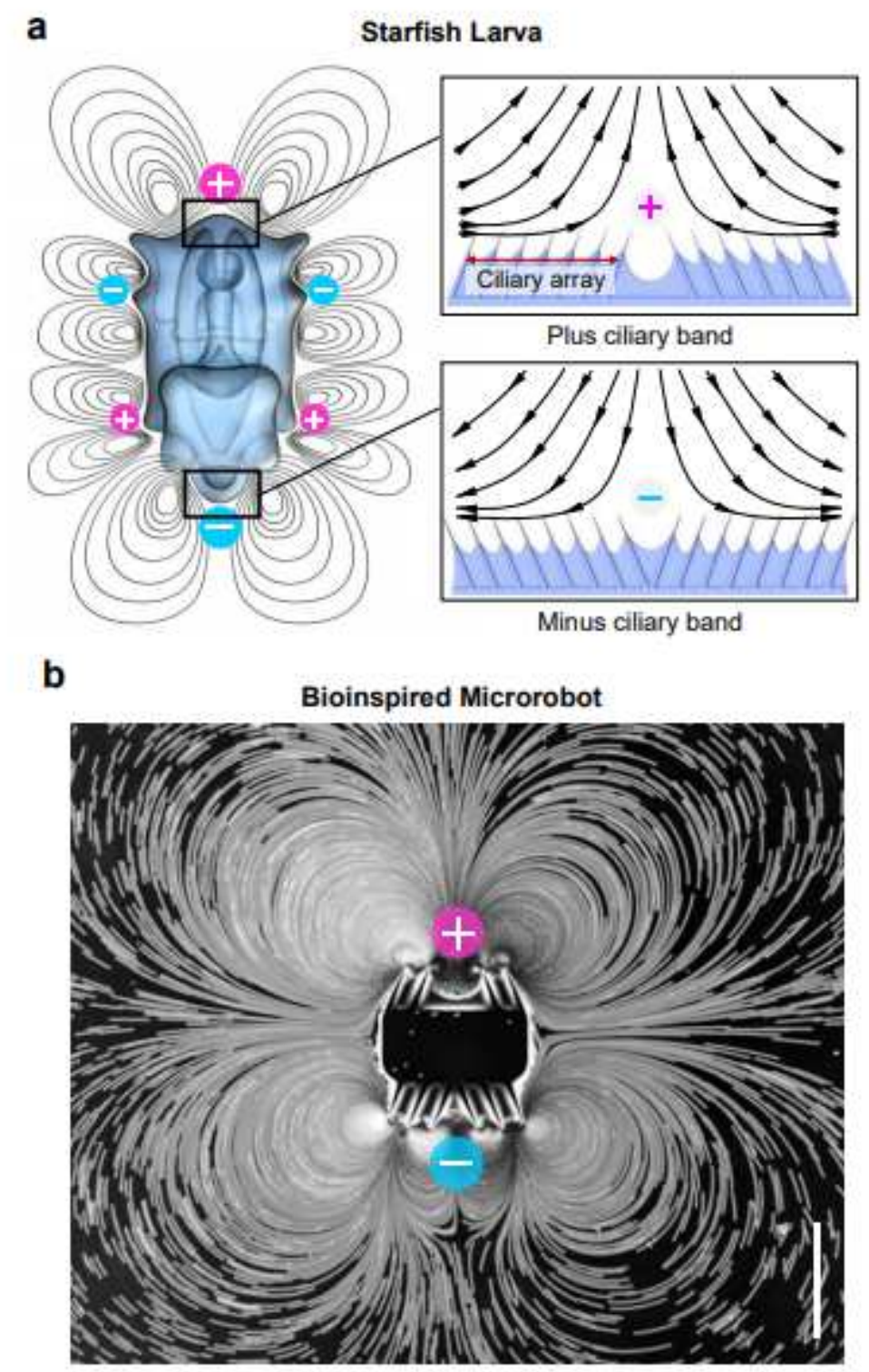

\section{Figure 1}

Starfish larva-inspired ultrasound ciliary band designs. a. A starfish larva exhibits a complex flow profile of counter-rotating vortices generated by series of + and - ciliary bands arranged on its body protuberances. Inset: (top) A + ciliary band comprises a pair of angled ciliary arrays that face each other and causes the liquid to flow away on a plane perpendicular to the centre of the ciliary band. (Bottom) A - ciliary band consists of two ciliary arrays oriented away from each other and directs the liquid flow to the centre of the band. b. A starfish-inspired microrobot consisting of a + (top) and a - (bottom) ciliary band placed in a tracer solution. The ciliary bands oscillate when actuated by ultrasound, producing complex flow profiles similar to those observed with its biological counterpart. Scale bar, $250 \mu \mathrm{m}$. 


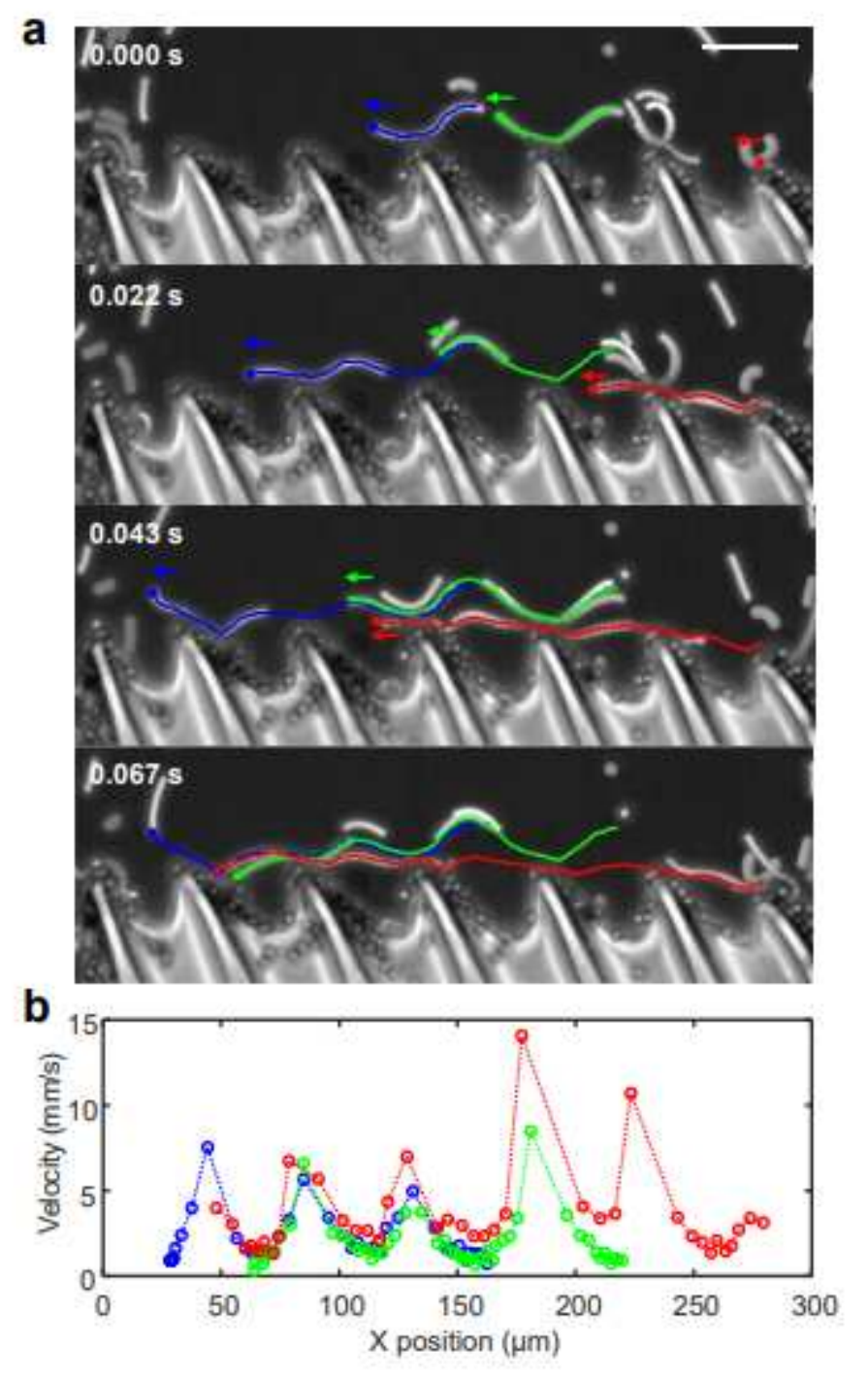

\section{Figure 2}

Tangential flow along angled ciliary array when exposed to ultrasound. a. Image sequences demonstrate $\sim 6 \mu \mathrm{m}$ tracer particles, indicated by red, green, and blue lines, travelling along one cilium tip to the next from right-to-left in the direction the tips are angled at excitation frequency and amplitude of $33.7 \mathrm{kHz}$ and 5 VPP, respectively (see also Movie S1). b. Velocity analysis of the tracers revealed a cyclic acceleration/deceleration pattern. Particles reached maximum speeds when they approached a ciliary tip, followed by a deceleration phase. Scale bar, $50 \mu \mathrm{m}$. 
a

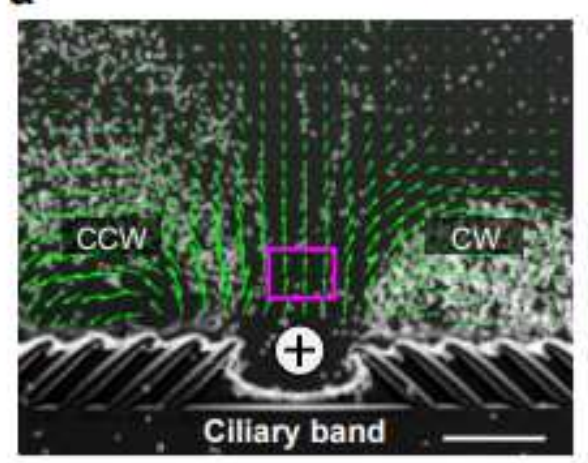

b

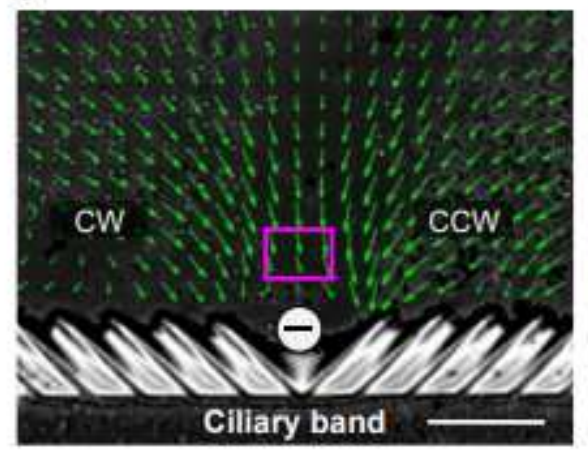

C

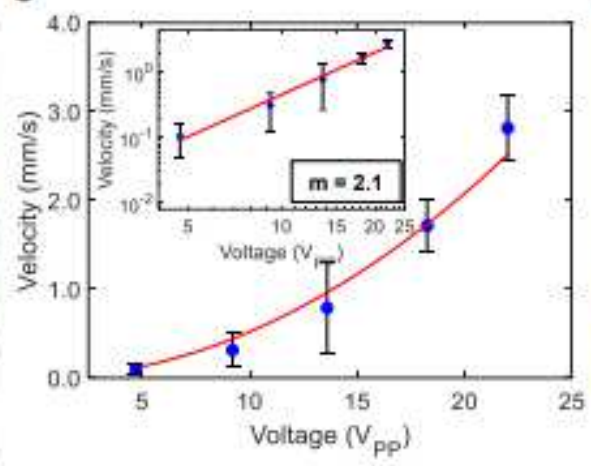

d

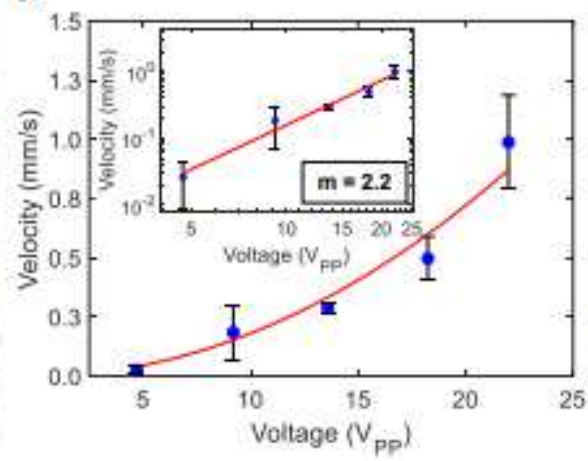

e

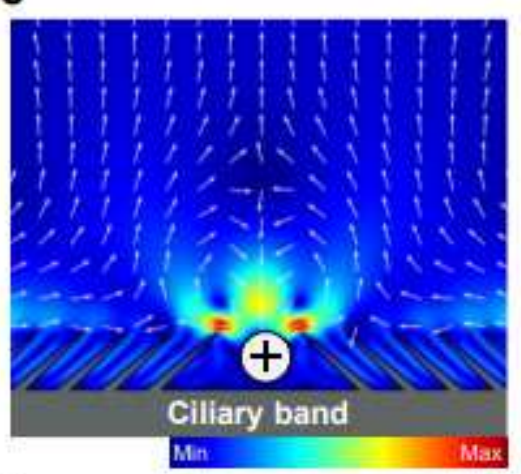

f

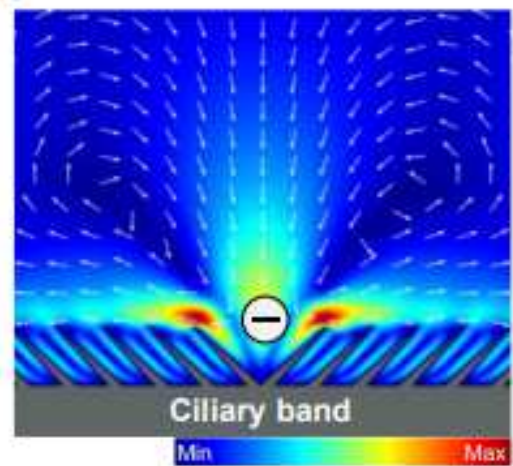

\section{Figure 3}

Experimental, characterization, and numerical demonstration of bioinspired ultrasound ciliary bands (Movie S2). a. PIV generated velocity fields of a + ciliary band that causes the liquid to flow away on a plane perpendicular to the centre of the ciliary band at excitation frequency and amplitude of $68.7 \mathrm{kHz}$ and 20 VPP, respectively. b. PIV generated velocity fields of a - ciliary band, which directs the liquid flow to the centre of the band at $68.8 \mathrm{kHz}$ and $22 \mathrm{VPP}$, respectively. Plots of average velocities of c. + and d. ciliary bands at sites indicated by magenta boxes in $a$. and $b$. versus voltage applied. The particles immersed in water transport at a speed proportional to the square of the voltage applied and this quadratic relation is reasonably well satisfied as indicated by the log plots. In each data point, 400 velocity measurements are averaged and the standard deviation is calculated and they are represented as black error bars. Numerical simulation of microstreaming using perturbation approach of e. + ciliary band and f. - ciliary band. Scale bar, $100 \mu \mathrm{m}$. 
a

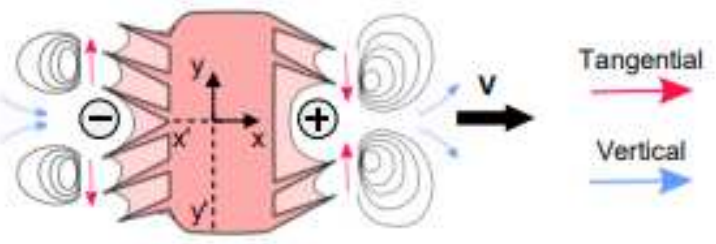

C

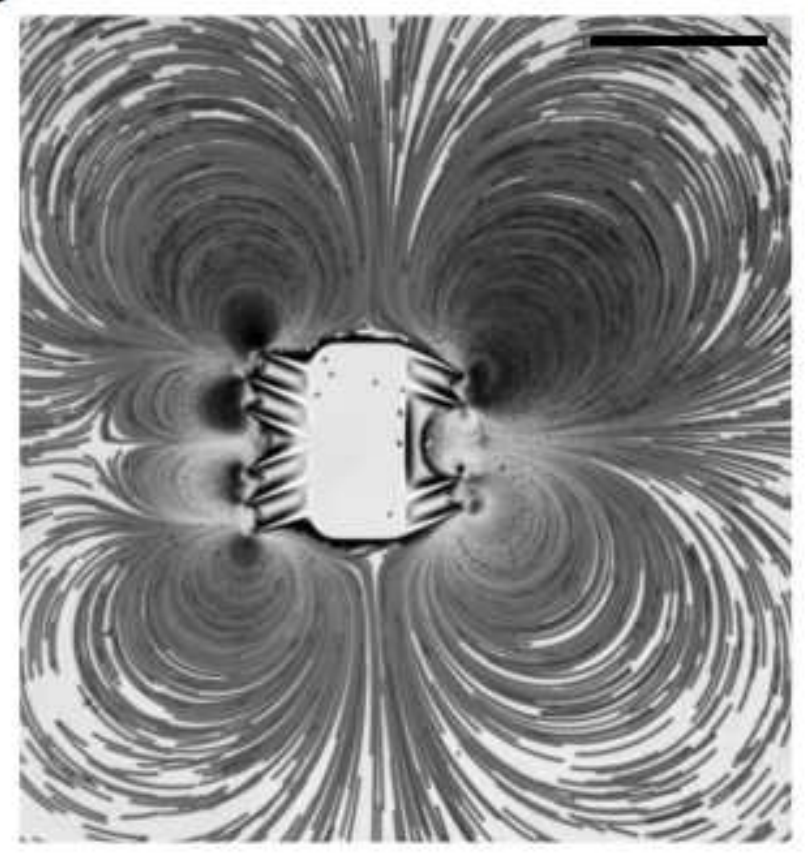

b

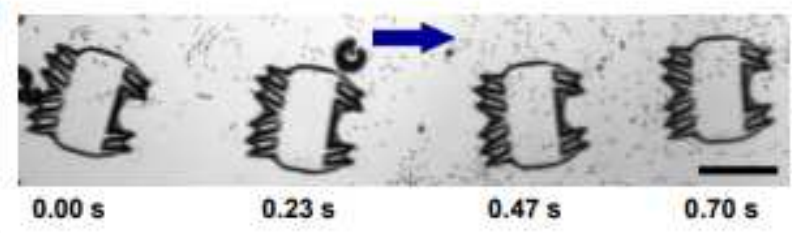

d

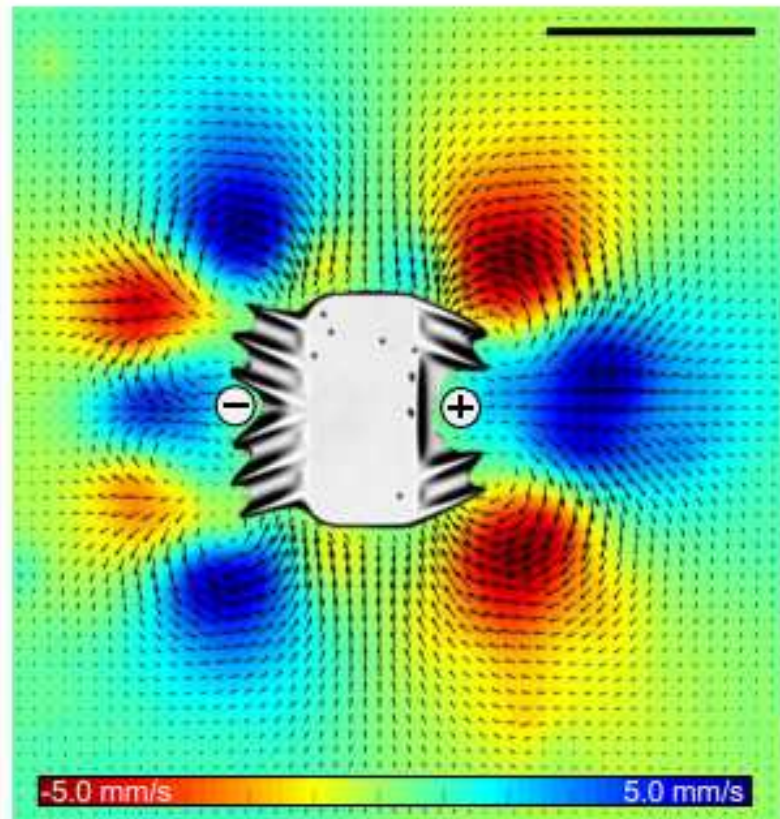

\section{Figure 4}

Propulsion of bioinspired ultrasound microrobot. a. Schematic of an artificial microrobot consisting of a - ciliary band on the left and a + ciliary band on the right. Red and blue arrows indicate tangential and vertical velocity, respectively. b. Superimposed time-lapse image of controlled translation motion of a microrobot at excitation frequency and amplitude of $68.8 \mathrm{kHz}$ and $20 \mathrm{VPP}$, respectively (see also Movie S4). c. Image sequence illustrating the streaming flow profile of the bioinspired microrobot. A stack of 150 images was used; the video was captured using a high-speed camera at a framerate of $1069 \mathrm{fps}$ (see also Movie S5). d. PIV generated velocity fields demonstrate the complex flow behaviour developed in the surrounding liquid. Minimum and maximum flow velocities are marked by red and blue regions, respectively. Scale bars, $250 \mu \mathrm{m}$. 
a

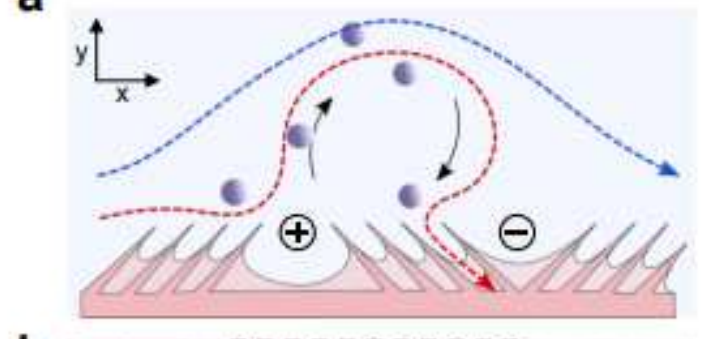

b

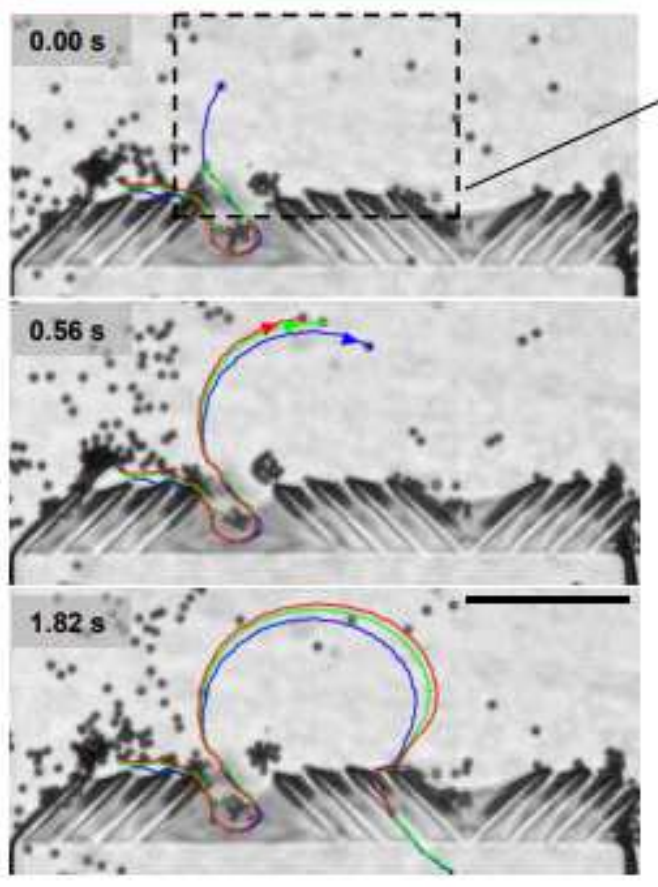

C

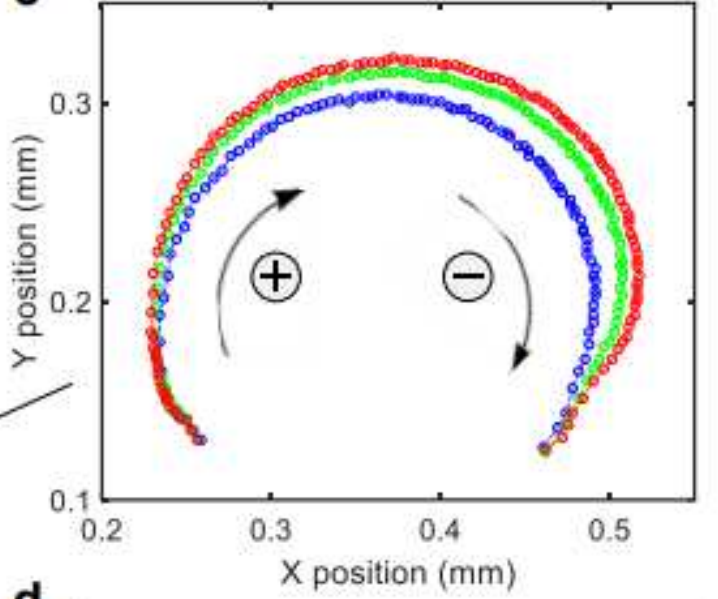

d

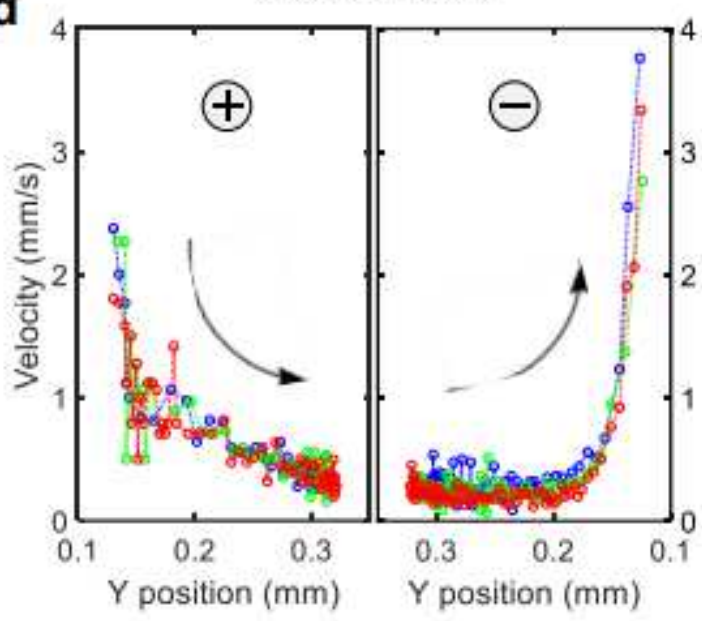

Figure 5

Bioinspired microparticle trapping using a combination of + and - ciliary bands. a. Schematic of a bioinspired trapping mechanism consisting of a + ciliary band adjacent to a - ciliary band (see also Movie S7). b. Image sequence demonstrating microparticles becoming trapped in the - ciliary band, as indicated by red, green, and blue trajectories at $68.5 \mathrm{kHz}$ and 20 VPP. c. Expanded spatial trajectories of microparticles that became trapped by the flow field produced from the adjacent + and - ciliary bands. $d$. Plots of microparticle velocity versus yposition: the left panel indicates the source and the right panel the sink behaviour of the trapping ciliary band. Scale bar, $200 \mu \mathrm{m}$.

\section{Supplementary Files}

This is a list of supplementary files associated with this preprint. Click to download.

- StarfishLarvaeSupplementaryFinalCD.docx

- Movies1.mp4

- MovieS2.mp4

- MovieS3.mp4 
- MovieS4.mp4

- MovieS5.mp4

- MovieS6.mp4

- MovieS7.mp4 